\title{
Esther Benbassa, Jean-Christophe Attias (éds.), $L a$ haine de soi. Difficiles identités
}

Bruxelles, Éditions Complexe, 2000, 306 p. (bibliogr.)

Joëlle Allouche-Benayoun

\section{OpenEdition}

Édition électronique

URL : http://journals.openedition.org/assr/2512

DOI : $10.4000 /$ assr.2512

ISSN : $1777-5825$

Éditeur

Éditions de l'EHESS

Édition imprimée

Date de publication : 1 octobre 2004

Pagination : 53-158

ISBN : 2-222-96754-6

ISSN : 0335-5985

\section{Référence électronique}

Joëlle Allouche-Benayoun, «Esther Benbassa, Jean-Christophe Attias (éds.), La haine de soi. Difficiles identités ", Archives de sciences sociales des religions [En ligne], 128 | octobre - décembre 2004, document 128.51, mis en ligne le 18 novembre 2005, consulté le 24 septembre 2020. URL : http:// journals.openedition.org/assr/2512 ; DOI : https://doi.org/10.4000/assr.2512 
comme un moyen de communication globale, " un langage qui utilise les mots et les gestes, l'expression verbale et corporelle » (p. 94), qui n'exclut en rien la parole, et reconnaît par ailleurs la place fondamentale de la prédication au cours du culte, comme outil d'évaluation pour les paroissiens et instrument d'innovation pour le pasteur.

Et c'est finalement lorsque l'A. s'éloigne de la description par les sens que l'analyse devient intéressante, notamment lorsqu'il étudie toujours dans la troisième partie - l'océanisation de la cène, qui consiste à remplacer le pain et le vin par des éléments locaux comme la noix de coco et le 'uru (fruit de l'arbre à pain).

L'A. dit du rite qu'il est un événement qui, tout en se répétant, garde un caractère extraordinaire et c'est pour conserver ce caractère extraordinaire qu'il doit sans cesse s'adapter, innover. En introduisant dans le processus même de ritualisation une dimension dynamique, l'A. rompt avec une lecture en termes antagonistes des rapports entre évangile et culture polynésienne qui sous-entend que l'Église s'éloigne de l'évangile à mesure qu'elle se rapproche de la culture polynésienne. L'A. prend ainsi le contre-pied de ceux qui estiment que cette théologie contextuelle polynésienne est « illusoire et dangereuse » dans la mesure où « elle défendrait un idéal polynésien qui n'a jamais existé. Elle "sacraliserait" la culture polynésienne. Elle conduirait même au pire nationalisme » (p. 67) et propose au contraire une lecture compréhensive qui apporte des éclairages théologiques intéressants, en insistant sur la dimension communautaire et pédagogique de ces cérémonies tout à la fois protestantes et polynésiennes.

\section{Gwendoline Malogne-Fer.}

BEDOYÈRE (Quentin de la).

Autonomy and Obedience in the Catholic Church. The Future of Catholic Moral Leadership. Londres-New York, $\mathrm{T} \& \mathrm{~T}$ Clark, 2003, 165+IX p. (bibliogr., index).

L'auteur est cadre dans le monde des affaires et conseiller matrimonial. C'est à la jonction de ce double titre qu'il nous livre un essai sur l'autorité morale catholique. Il plaide pour le respect de la liberté de conscience et sa conséquence, la nécessité de modifier la gestion du discours moral, en particulier quand les autorités romaines sont en cause.

Dans un style clair et alerte, l'A. rappelle la tradition catholique, spécialement en ce qui concerne les discussions sur l'autonomie de la conscience individuelle, son rapport à l'autorité ecclésiastique et le principe de subsidiarité de cette dernière. Sur chacun de ces points, définis avec soin, il confronte les courants issus du Concile du Vatican II et le management de la morale sous Paul VI et Jean-Paul II. Quant à la réception, son analyse factuelle est toute centrée sur la situation anglaise, et elle s'appuie sur des études américaines ou britanniques.

Le propos fait état des aspects psychosociaux et religieux institutionnels de la souveraineté de la conscience, avant d'enchaîner sur la subsidiarité, à savoir le partage des pouvoirs entre les différents niveaux du gouvernement catholique, et sur la participation de laïcs à la prise de décision. Il en va de la recrédibilisation sociale d'une institution confrontée aux exigences du management moderne sans pour autant devoir évacuer les acquis de la tradition. De ce point de vue, nous sommes devant la forme sociale au sens de Georg Simmel, c'est-àdire une création collective mettant en interaction des exigences opposées.

Le sociologue saura gré à l'A. d'avoir évité de psychologiser indûment son argument et d'avoir apporté des précisions historiques ou conceptuelles sur des points trop souvent méconnus, tels la subsidiarité et sa légitimation, l'autonomie de la conscience et ses conditions relationnelles. On aura compris que l'analyse critique de la dynamique catholique est d'ordre interactionniste et non structuraliste.

\section{Paul-André Turcotte.}

BENBASSA (Esther), ATTIAS (Jean-Christophe), éds.

La Haine de soi. Difficiles identités. Bruxelles, Éditions Complexe, 2000, 306 p. (bibliogr.).

Cet ouvrage rassemble les contributions d'historiens, de psychanalystes, de psychiatres, de sociologues, d'anthropologues et de philosophes à un colloque qui s'est tenu en avril 1998 à la Sorbonne.

C'est Theodor Lessing, un intellectuel juif allemand, qui forgea ce concept de haine de soi en 1930, à travers son ouvrage La Haine de soi juive : à une époque où l'antisémitisme touchait toutes les sociétés européennes, ce « phénomène morbide » servit d'abord « à penser la psychopathologie de certaines franges du peuple juif, intériorisant parfois jusqu'au suicide, le regard de rejet qui se portait sur elles ». Ce phénomène touche-t-il les mêmes et/ou d'autres aujourd'hui ? L'apport le plus intéressant de ce travail, par ailleurs d'une facture classique sur un tel sujet, est de mettre l'accent, à la suite de 
Lessing lui-même, sur l'universalité du phénomène.

L'ouvrage se décompose en sept parties, qui chacune explore un aspect de cette haine. Les trois premières parties ouvrent sur des interrogations : la Maladie d'un peuple?, Une Maladie de l'âme ?, Existe-t-il une homophobie gay?

La première partie reprend pour l'essentiel la démarche de Lessing lui-même : la haine de soi est universelle, ce n'est pas $L a$ maladie du peuple juif, et le cas juif n'est qu'un cas parmi d'autres. Mais quel cas... l'analyse par Jacques le Rider de Otto Weininger, ce jeune philosophe juif allemand que le refus de son identité juive a poussé jusqu'au suicide à 23 ans est une magistrale démonstration de ce cas par excellence de « haine de soi juive». Psychiatre et psychanalystes s'attachent ensuite à nous montrer comment s'élabore dès l'enfance, un certain refus de soi, qui culmine en quelque sorte chez les anorexiques, qui haïssent aussi dans leur corps, la preuve matérielle de leur identité sexuelle. Haine de soi repérable chez certains homosexuels, à Saint-Germain-des-Prés, ou à Istanbul, qui stigmatisent à leur tour ceux d'entre eux qui ne seraient pas conformes à ce qu'ils décrètent être la bonne norme homosexuelle.

Plus politique, la quatrième partie explore les haines, de soi et des autres, porteuses de «violences et d'exclusions », dans l'Iran islamiste, le Cambodge des khmers rouges, et dans nos sociétés occidentales à travers la figure du sans-abri.

Les cinquième, sixième et septième parties présentent des trajectoires individuelles, et s'interrogent sur le devenir du «soi». «Se perdre ou se retrouver dans l'écriture », et renoncer au «soi », renouent avec la problématique de départ, en nous présentant les itinéraires pleins de souffrance, de mal nés et mal aimés. Cela vaut pour les juifs : Maurice Sachs dont la haine de soi atteignit son apogée avec son enrôlement dans la Gestapo en 1943, ou Simone Weill envers qui l'on « reste interdit devant le fait que...(elle) qui compatit à toute la souffrance du monde, n'écrit pas une ligne sur les persécutions et les massacres des juifs, alors qu'elle est à Londres, où.... parviennent de nombreuses informations concernant l'extermination des juifs » (Martine Leibovici). Cela vaut aussi pour les non juifs: un texte de Hervé Poutet s'attache ainsi à analyser dans « haine de soi et filiation : la rédemption par l'écriture » les textes de l'écrivain Michel del Castillo qui dit de lui-même : «j'ai mal à l'enfance » alors que Maya Burger tente de discerner dans certaines expériences mystiques en Inde, ce qui pourrait relever de cette catégorie conceptuelle.

«Le "soi” recomposé » clôt l'ouvrage sur deux contributions mettant à nouveau en scène des expériences juives, que ce soit celles du « changement de nom: le signe, la haine, le soi » à travers l'analyse des changements de patronymes juifs en Allemagne en 1933 et en France en 1945, ou celles concernant « conversion et Haine de soi » où est analysée à travers la littérature rabbinique, l'image pleine d'ambivalence du converti au judaïsme chez les juifs.

Ce sur quoi donne à réfléchir ce très riche ouvrage, c'est bien entendu la question de l'Autre, de sa place dans la construction individuelle, dans l'économie psychologique de chacun. Et encore que cela ne soit pas son objectif explicite, l'ouvrage donne à réfléchir sur l'actualité la plus brûlante.

Joëlle Allouche-Benayoun.

128.52

BREGER (Marshall J.), ed.

The Vatican-Israel Accords. Political, Legal, and Theological Contexts. Notre Dame (Indiana), University Notre Dame Press, 2004, 392 p. (index).

La signature, le 30 décembre 1993, entre le Saint-Siège et l'État d'Israël d'un Accord fondamental, correspondait à un changement majeur de position du Saint-Siège en regard des problèmes du Proche-Orient, mais en même temps ouvrait des perspectives nouvelles dont, dans un premier temps, l'établissement de relations diplomatiques normales entre le Vatican et Israël. Pour le dixième anniversaire de cet événement, l'auteur, professeur de droit à la Columbus School of Law de l'Université catholique d'Amérique, a voulu faire le point et proposer, ce qui n'avait pas été fait jusqu'à présent de façon systématique, une analyse historique, juridique et théologique de l'Accord.

Après une rapide présentation du déroulement de la négociation (Lorenzo Cremonesi), et une analyse de la signification de l'Accord, du point de vue du Saint-Siège (David-Maria A. Jaeger, o.f.m.), dans le contexte de l'évolution de l'Église après le Concile Vatican II (Silvio Ferrari) et du point de vue de l'État d'Israël (Leonard Hammer), l'ouvrage traite de l'Accord en regard, d'une part, de la pratique vaticane des Concordats et, d'autre part, de la Loi internationale. Ainsi, sont présentées, dans une troisième partie, la position théorique et pratique de l'Église catholique à propos des Concordats (Roland Minnerath), ou une comparaison de l'Accord avec le Concordat espagnol (Rafael 